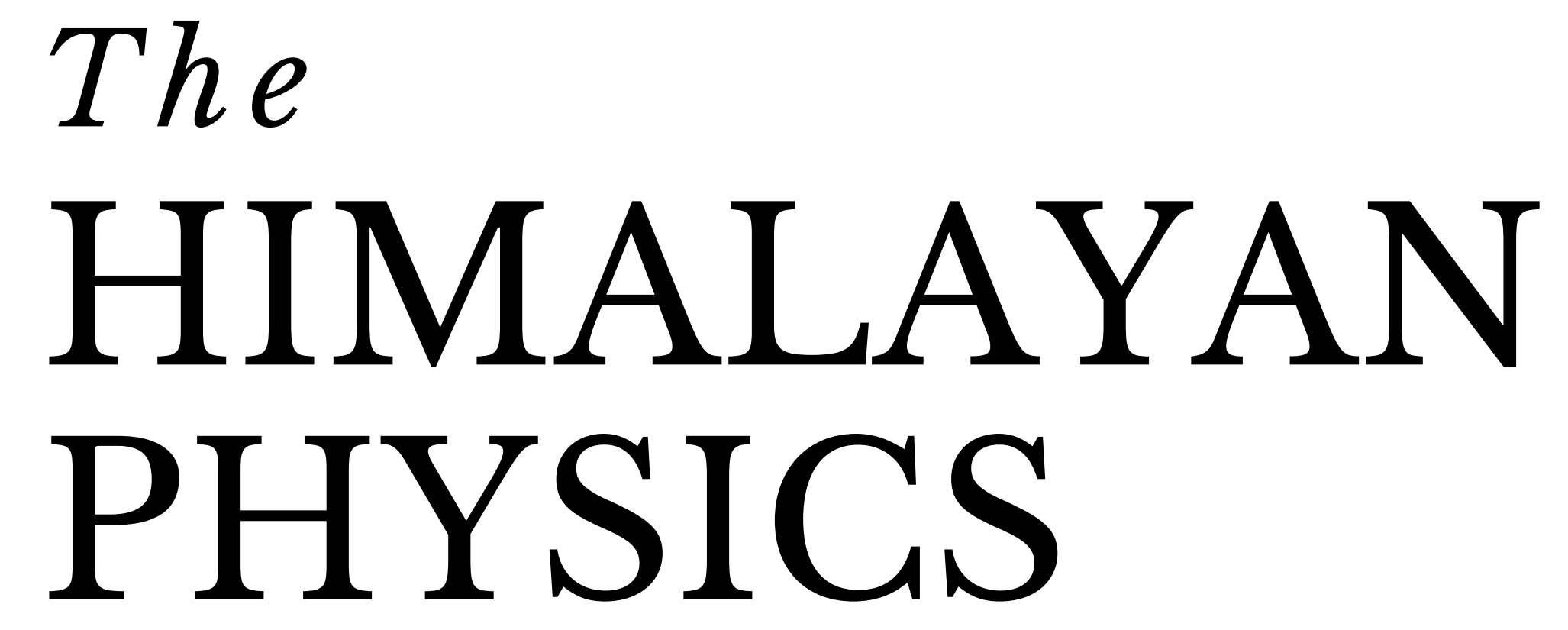

A peer-reviewed Journal of Physics

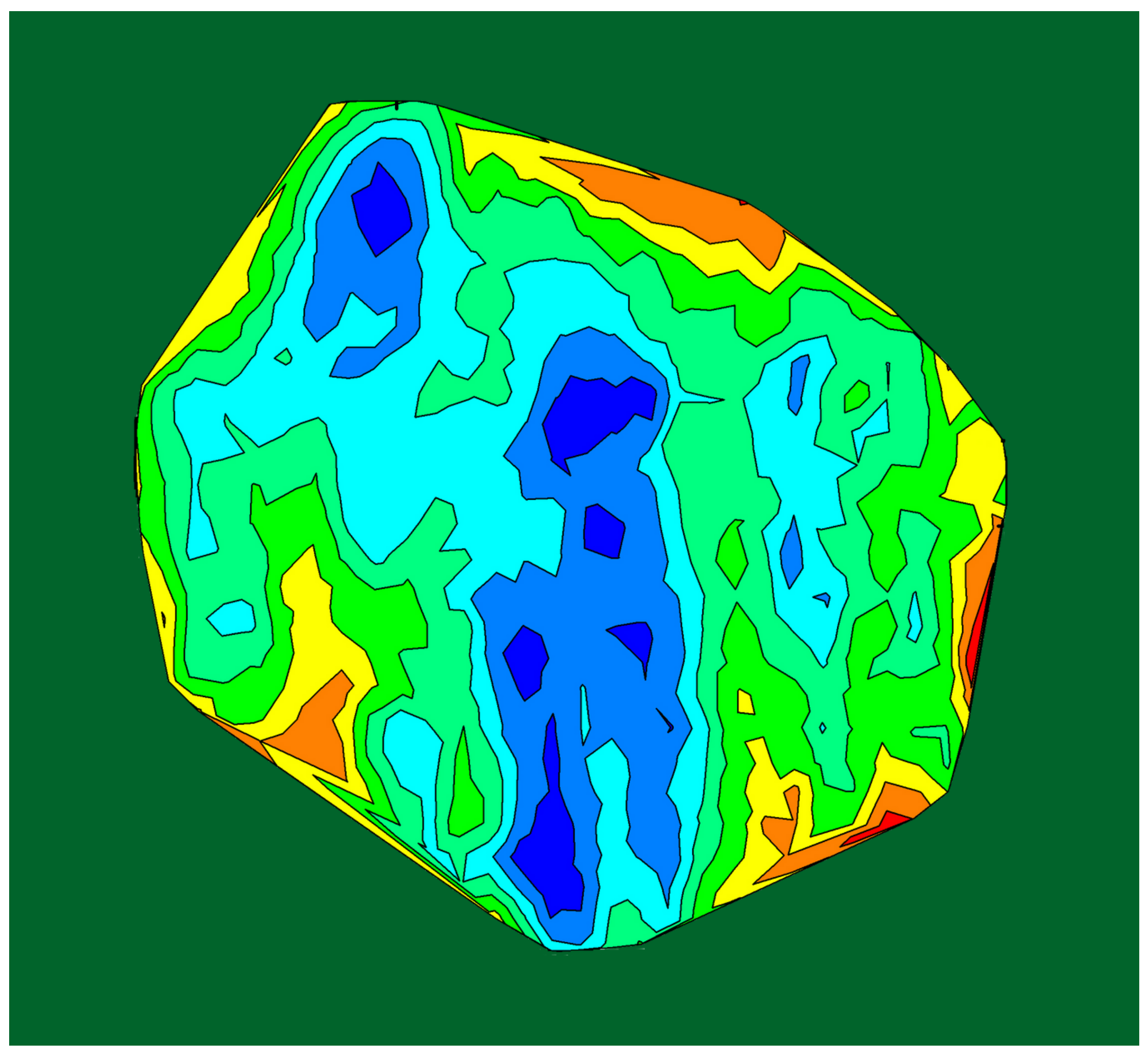

Department of Physics, Prithvi Narayan Campus, Pokhara Nepal Physical Society, Western Chapter, Pokhara 


\section{Publisher}

Department of Physics, Prithvinarayan Campus, Pokhara Nepal Physical Society, Western Chapter, Pokhara

\section{The Himalayan Physics}

Volume 9, December 2020

ISSN 2542-2545

The Himalayan Physics (HimPhys) is an open access peer-reviewed journal that publishes quality articles which make innovative contributions in all areas of Physics. HimPhys is published annually by Nepal Physical Society (Western Regional Chapter), and Department of Physics, Prithvi Narayan Campus, Pokhara. The goal of this journal is to bring together researchers and practitioners from academia in Nepal and abroad to focus on advanced techniques and explore new avenues in all areas of physical sciences and establishing new collaborations with physics community in Nepal.

\section{Chief Editor}

Kapil Adhikari

\section{Associate Editor \\ Aabiskar Bhusal}

(C)2020, Publishers. All rights reserved.

This publication is in copyright. Subject to statutory exception and to the provisions of relevant collective licensing agreements, no reproduction of any part may take place without written permission of the publishers.

Cover: Contour map of dust mass. (C) Mijas Tiwari. Printed from article in the current issue, with permission. 


\section{The HIMALAYAN PHYSICS}

A peer-reviewed Journal of Physics

\section{Chief Editor}

Kapil Adhikari

Associate Editor

Aabiskar Bhusal

\section{Publisher}

Department of Physics, Prithvi Narayan Campus, Pokhara Nepal Physical Society, Western Chapter, Pokhara 


\section{Nepal Physical Society Western Regional Chapter Pokhara, Nepal}

\section{President}

Min Raj Lamsal

Immediate Past President

Jeevan Regmi

Vice-President

Sundar Prasad Dhakal

Secretary

Ravi Karki

Treasurer

Dipak Adhikari

Joint Secretary

Sujan Lamsal

Editorial Member

Kapil Adhikari

Members

Amrit Dhakal

Laxman Thapa

Laxman Timilsina

Narayan Prasad Bhandari

Pradeep Subedi

Advisory Board

Prof. Dr. Pradip K. Bhattarai

Pabitra Mani Poudyal

Surya Bahadur G.C.

Parashu Ram Poudel

Prof. Dr. Shovakanta Lamichhane

Kul Prasad Dahal

Dr. Krishna Raj Adhikari

Ram Sajile Verma 


\section{Himalayan Physics Vol-9 (2020)}

\section{TABLE OF CONTENTS}

Metal Organic Frameworks(MOFs) as efficient carrier for targeted nanodrug delivery

R. Karki, D. Adhikari, K. Adhikari, N. Pantha

A Density Functional Theory Study on Paracetamol-Oxalic Acid Co-Crystal

P. Paudel, K.R. Adhikari, K. Adhikari

First-principles study of $\mathrm{C}$ cites vacancy defects in water adsorbed graphene

H.K. Neupane, N.P. Adhikari

Diusion of fructose in water: a molecular dynamics study

S. Bhusal, N. Pantha

Study of aecting factors of meteorological parameters on solar radiation on Pokhara P.M. Shrestha, J. Regmi, U. Joshi, K.N. Poudyal, N.P. Chapagain, I.B. Karki

Variation of mean value of velocity of ion with dierent obliqueness of magnetized plasma sheath

B.R. Adhikari, H.P. Lamichhane, R. Khanal

Study of dust properties of two far infrared cavities nearby asymptotic giant branch stars under infrared astronomical satellite maps

M. Tiwari, S.P. Gautam, A. Silwal, S. Subedi, A. Paudel, A. K. Jha

An experimental study on irradiated interface of silicon

M.R. Lamsal

Calculation of energy loss of proton beam on thyroid tumor

K. Giri, B. Paudel, B.R. Gautam

Study of noise level status at dierent rice mills in Surkhet Valley, Nepal

D.R. Paudel, H.N. Baral

Elliptically polarized laser assisted elastic electron-hydrogen atom collision and differential scattering cross-section

K. Yadav, S.P. Gupta, J.J. Nakarmi

Geodynamics of Gorkha earthquake (Mw 7.9) and its aftershocks

R.K. Tiwari and H. Paudyal 


\title{
Calculation of energy loss of proton beam on thyroid tumor
}

Research Article

\author{
K. Giri ${ }^{1} *$ B. Paudel $^{1}$, B.R. Gautam ${ }^{2}$ \\ 1 Department of Physics, Tri-Chandra Multiple Campus, Kathmandu, Nepal \\ 2 Department of Chemistry, Physics and Materials Science, Fayetteville State University, North Carolina, USA
}

\begin{abstract}
Proton beam therapy is an emerging technique in radiotherapy. The Bragg peak of a proton enables it to lose most of its energy to the targeted tissue like tumor cells, with less impact of healthy tissues and organs. This property of a proton beam makes it ideal for clinical applications. When organ safekeeping is our priority then proton beam therapy is the most effective tool to damage nearby affected tissues. For efficient treatment planning in thyroid tumor, the maximal energy loss of proton beam in its tissues must be exactly calculated. The method of computer simulation is employed for the calculation of energy loss by energized proton beam irradiation on thyroid tumor at a depth of $22 \mathrm{~mm}$. The stopping power and range data agrees with standard reference data. Of the $50 \mathrm{MeV}$ energy of proton beam, the most of the energy is absorbed on various layers viz. skin, adipose tissue, skeletal muscles and thyroid which are approximately $1.5 \mathrm{MeV}, 5.3 \mathrm{MeV}, 10.5 \mathrm{MeV}$ and $33.5 \mathrm{MeV}$ respectively. In total $99.96 \%$ of energy of proton beam is absorbed by the targeted tissues.
\end{abstract}

Keywords: : Proton beam therapy • Thyroid • Energy loss $\bullet$ Stopping power $\bullet$ Biological targets

\section{Introduction}

The beam of proton loses its energy while traversing the medium. When it passes through the living tissues it causes damage to it. Nowadays beams of heavy charged particles specifically protons and ${ }^{6} C_{12}$ ions are employed for the clinical applications in radiotherapy [1]. And a highly effective tumor treatment method called hadron therapy has been developed. It is a type of hadron therapy where particle accelerator is used to target an affected with a beam of protons [2]. This decreases the probability of recurrence of tumor. Robert Wilson in 1946 proposed to use accelerator-produced beams of protons to treat deep-seated tumors in human [3]. These charged particles lose their energy to target tissue causing damage to the DNA of cells, ultimately killing them or stopping their reproduction. Cancerous cells are particularly susceptible to attacks on DNA because of their high rate of division and their reduced abilities to repair DNA damage. Some cancers with specific defects in DNA repair may be more sensitive to proton radiation [4]. For efficient tumor treatment, we need to know two things: how the charged particles like proton interact with biological matter and how big amount of energy is

\footnotetext{
* Corresponding Author: karangiri575@gmail.com
} 
deposited during passing into tissues which give information about a dose that patient received during therapy. Because experiments on animals or humans tissues are rather complicated, we need to create a simple model (or theoretical program) that could be used to calculate the dose distributions in biological matter [5]. For this purpose, a computer program, SRIM is used, which is usually used in the calculation of energy loss by nuclear physicist.

\section{Thyroid}

The thyroid gland or simply the thyroid is an endocrine gland in the neck, consisting of two lobes connected by an isthmus. It is found at the front of the neck, below the Adams apple [6]. The infrahyoid muscles attached to hyoid bone lie in front of the gland and the sternocleidomastoid muscle to the side. Both are skeletal muscles [6]. The trachea, larynx, lower pharynx and esophagus all lie behind the thyroid [7]. If the thyroid gland is radiated, it may lead to hypothyroidism, a glandular condition which creates symptoms such as reduced metabolism, sluggishness, high cholesterol, weight gain, and depression. A study on Craniospinal Irradiation using conventional radiotherapy versus proton therapy found that proton beam therapy reduces the risk of hypothyroidism significantly [8].

\section{Stopping Power and Range}

The charged particles interact with matter mainly in two ways it is either electronic interaction or nuclear interaction. Electronic interaction is when the projectile particle interacts with the electrons of the atoms in the target medium that leads ionization. And the nuclear interaction causes production of secondary particles or simply the vibration. In both interaction certain amount of energy is lost and causes the particles to slow down [9]. The energy loss per unit length of the medium $\left(\frac{d E}{d x}\right)$; energy gradient is known as stopping power of the medium. It can be calculated by using Bethe Bloch formula [10];

$$
-\frac{d E}{d x}=\left(\frac{e^{2}}{4 \pi \epsilon_{o}}\right)^{2} \frac{4 \pi N_{A} Z \rho}{m c^{2} \beta^{2} A}\left[\ln \left(\frac{2 m c^{2} \beta^{2}}{I}\right)-\ln \left(1-\beta^{2}\right)-\beta^{2}\right]
$$

Here, the minus sign signifies the energy loss, $\epsilon_{o}$ is the vacuum permittivity, $e$ is electron charge, $m$ is rest mass, $N_{A}$ is Avogadro number, $Z$ is atomic number, $A$ is atomic mass number, $\rho$ is density of the medium, $\mathrm{I}$ is excitation potential and $\beta=\frac{v}{c}$, $v$ being velocity of particle, $c$ the velocity of light.

The distance $x$ is not always expressed in meters, but often in units of mass per square meter. The distance travelled by the charged particle before it loses its all energy is known as its range. As the energy loss is a statistical phenomenon there are certain variations in the range which we call the range straggling. Range can be determined from the stopping power provided that we know the form of $\mathrm{S}$ from zero energy up to the initial energy of the particles in the incident beam [10].

$$
R(E)=\int_{0}^{E}\left(\frac{d E^{\prime}}{d x}\right)^{-1} d E^{\prime} \approx \sum_{0}^{E}\left(\frac{d E^{\prime}}{d x}\right)^{-1} \Delta E
$$


Here, $E$ is the ion's initial kinetic energy and the summation denotes that the continuous transport is approximated by calculations of discrete steps.

\section{Materials and Method}

A computer simulation process (SRIM 2013) is employed when human neck region is exposed to beam of proton targeting thyroid tumor at a depth $21 \mathrm{~mm}$ from skin. At first, energy required is calculated using the stopping and range table for water samples and then a multi-layer target was built on the TRIM section. Simulation was run for 100000 protons. From the data obtained from TRIM calculation, various aspects of energy loss such as energy loss in ionization and phonon and energy loss in different layers and final distribution of protons in the target were calculated. Graphs were plotted using MATLAB. Different layer properties is shown in Table 1.

\begin{tabular}{lcc} 
Table 1. Various layer's properties of body \\
\hline Layer Name & $\begin{array}{c}\text { Layer Width } \\
(\mathrm{mm})\end{array}$ & $\begin{array}{c}\text { Layer Density } \\
\left(\mathrm{g} / \mathrm{cm}^{3}\right)\end{array}$ \\
\hline Skin & 1.2 & 1.09 \\
Adipose tissue & 4.0 & 0.92 \\
Skeletal Muscle & 6.0 & 1.05 \\
Thyroid & 14.0 & 1.05 \\
\hline
\end{tabular}

SRIM is mainly used to conduct the experiment in ion implantation for the calculation of energy loss. It is a group of computer programs which calculate interaction of ions with matter which atomic and chemical compositions are also included in the compound dictionary bottom. SRIM is a program Transport of Ions in Matter (TRIM) is the main part of the SRIM. For the cancer cell treatment of various organ of human body TRIM calculation is the main calculation which gives the output when we give the certain amount of energy to the proton so we can call it the core of the SRIM. SRIM contains around 28,000 built-in experimental data for stopping power and therefore allows for accurate simulation of ion penetration into matter [11].

SRIM is based on a Monte Carlo simulation method, namely the binary collision approximation with a random selection of the impact parameter of the next colliding ion. Mainly SRIM contains input that is the ion to be implanted or ion to be used to irradiate tumor and the energy for the ion can be added by our desire.

The text data calculated by TRIM are organized in a spreadsheet using Microsoft excel and thus obtained data files are imported in MATLAB to obtain schematic graphs of related values.

\section{Results and Discussion}

The energy as calculated from stopping and range table was found to be $50 \mathrm{MeV}$. This is the energy if given to the proton beam it releases its maximum energy to the targeted range. The Fig. 1(a) reveals the deviation of 
our result from the standard one, PSTAR data.

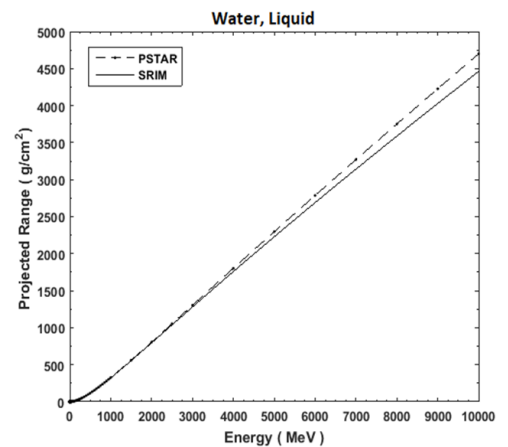

(a)

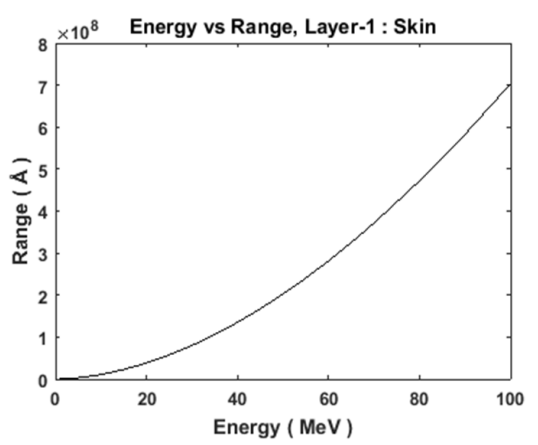

(b)

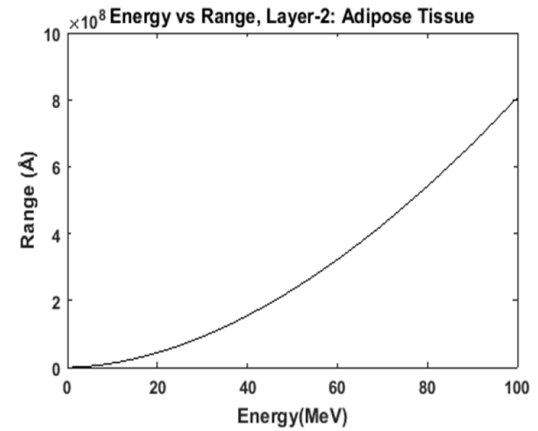

(c)

Figure 1. (a) Deviation of range from standard (PSTR), (b) Variation of range with energy in skin and (c) in adipose tissue

At lower energies of the proton beam the nature of the curve is consistent with the standard reference data obtained from PSTAR. The range versus energy graph is similar for various layers which show that the range of proton in biological target is a function of target density.

Figs. 1(b) and 1(c) show range of proton beam of high energy in the skin and adipose tissue. The range versus energy graph is similar for various layers which show that the range of proton in biological target is a function of target density. Figs. 2(c), 2(d) and 2(e) demonstrate the variation of energy gradient with ions energy in skin, adipose tissuse and skeletal tissue. The curves show that the the nuclear interaction have insignificant contribution on total stopping power of the medium, it is in fact almost 1/10000 times less than that due to electronic interaction. The major loss of energy is caused by electronic interaction of proton i.e. ionization. This is due to the smaller size of proton than the target atoms and a positive charge on it which attracts the electron out of its orbit. The stopping power seems to decrease as energy increases since high energy particle have high velocity which provide less time for interaction.

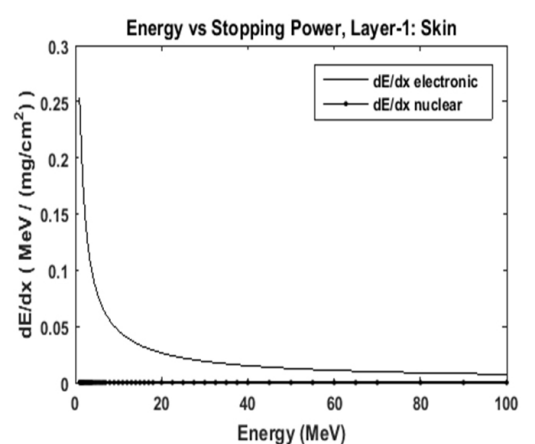

(c)

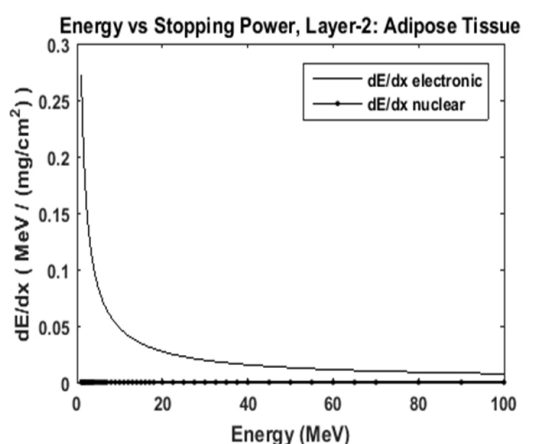

(d)

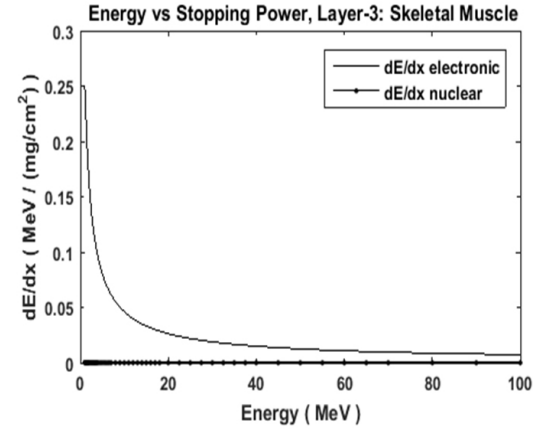

(e)

Figure 2. Variation of energy gradient with ions energy in (c) Skin, (d) Adipose Tissue, and (e) Skeletal Tissue

Fig: 3, shows the energy imparted to the target. The peak in the curve shows the maximum energy is 
imparted to the targeted cancerous cells results damaging of these cells with the minimum energy loss to the surrounding tissues. The data relating to Ions is the direct energy transferred from the protons to target electrons. The data relating to Recoils is the energy transferred from recoiling target atoms to the target electrons. This is also known as Bragg peak and it is seen at $21.22 \mathrm{~mm}$, which is the region of maximum energy loss. Area under the curve between any two points on the $\mathrm{x}$-axis gives the energy loss on that region.

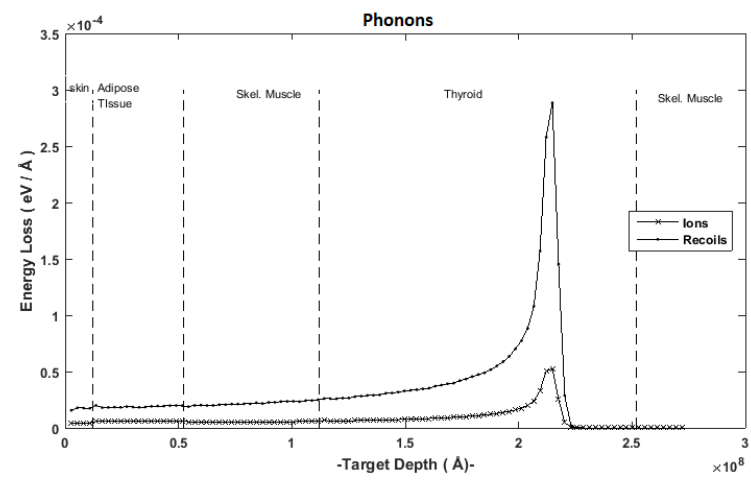

Figure 3. Variation of energy loss with target depth

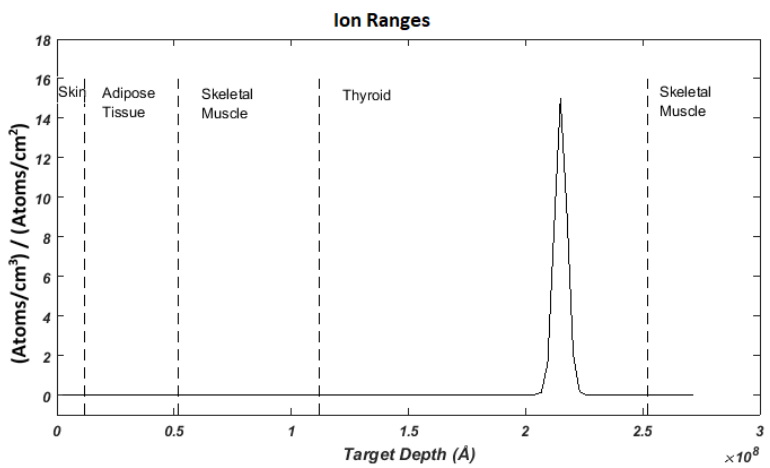

Figure 4. Proton range distribution.

Table 2 summarizes the energy loss on different layers due to ionization. Referring to the Fig. 4, the energy deposition is the maximum at the targeted site. At the end of the range and the ionization by recoil is negligible. A little discrepancy seen in the graph in adipose tissue layer is due to the relatively lower concentration of heavy elements like $\mathrm{Na}, \mathrm{Mg}, \mathrm{P}, \mathrm{Cl}$, and so forth.

Table 2. Energy loss on ionization

\begin{tabular}{lc}
\hline Depth $(\mathrm{mm})$ & Energy \\
\hline $0-1.36$ (Skin) & 1.490 \\
$1.36-5.44$ (Adipose tissue) & 5.252 \\
$5.44-11.42$ (Skeletal muscle) & 9.759 \\
$11.42-25.3$ (Thyroid) & 33.302 \\
$>25.3$ & 0 \\
\hline
\end{tabular}

\section{Conclusion}

We successfully determined the range in biological medium by using stopping and range table for liquid water which was consistent with SRD from PSTAR. TRIM data shows that $99.96 \%$ of the energy of proton is lost in the process of ionization by ion and $0.02 \%$ in ionization by recoils and only $0.02 \%$ energy is lost by production of phonon which is mainly by recoiling atoms. The energy deposited on various layers viz. skin, adipose tissue, skeletal muscle and thyroid are approximately $2.98 \%, 10.51 \%, 19.52 \%$, and $66.87 \%$ respectively, and protons scatter a little laterally in the tissue; the beam broaden less, stays concentrated on the tumor shape and delivers only low-dose side effects to nearby tissue. So we concluded that, water samples are useful in determination of 
range before treatment, thyroid tumor can be irradiated with proton beams causing less harm to healthy tissues and SRIM/TRIM codes are useful for qualitative analysis before the treatment.

\section{References}

[1] Khoroshkov V. Radiation beam therapy evolution: From X-rays to hadrons. Physics of Atomic Nuclei. 2006;69(10):1724-1742.

[2] Durante M, Cucinotta FA, Loeffler JS. Charged Particles in oncology. Frontiers in oncology. 2017;7:301.

[3] Wilson VC, McDonough J, Tochner Z. Proton beam irradiation in pediatric oncology: an overview. Journal of pediatric hematology/oncology. 2005;27(8):444-448.

[4] Liu Q, Ghosh P, Magpayo N, Testa M, Tang S, Gheorghiu L, et al. Lung cancer cell line screen links fanconi anemia/BRCA pathway defects to increased relative biological effectiveness of proton radiation. International Journal of Radiation Oncology, Biology, Physics. 2015;91(5):1081-1089.

[5] Maliszewska W, Sekowski P, Skwira-Chalot I. Application of instruments of nuclear physics to the calculation of theoretical dose distributions in various organs of the human body for beams used in hadrontherapy. Nukleonika. 2016;61(1):19-22.

[6] Standring S. Gray's anatomy:the anatomical basis of clinical practice. 40th ed. The address: The name of the publisher; 2008.

[7] Bogart BI, Ort V. Elsevier's integrated anatomy and embryology. Elsevier Health Sciences; 2007.

[8] Bielamowicz K, Okcu MF, Sonabend R, Mahajan A, Paulino AC, Adesina A, et al. Decreased incidence of hypothyroidism after craniospinal irradiation (CSI) with proton therapy (PRT) in patients with medulloblastoma. Neuro-Oncology. 2016;18(Suppl 3):iii161.

[9] El-Ghossain M. Calculations of stopping power, and range of ions radiation (alpha particles) interaction with different materials and human body parts. International Journal of Physics. 2017;5(3):92-98.

[10] Newhauser WD, Zhang R. The physics of proton therapy. Physics in Medicine \& Biology. 2015;60(8):R155.

[11] Ziegler JF, Ziegler MD, Biersack JP. SRIM-The stopping and range of ions in matter (2010). Nuclear Instruments and Methods in Physics Research Section B: Beam Interactions with Materials and Atoms. 2010;268(11-12):1818-1823. 